\title{
TRATAMENTO COMPUTACIONAL DA TOPOLOGIA DE GRANDES REDES ELÉTRICAS
}

\author{
M. Th. Schilling* \\ T. M. L. Assis* \\ D. S. T. Cárdenas* \\ R. P. D. Ross $^{\dagger}$ \\ *Universidade Federal Fluminense \\ Departamento de Engenharia Elétrica / Instituto de Computação \\ Rua Passo da Pátria, 156, Bloco D, sala 509, \\ CEP 24210-240 - Niterói - RJ \\ ${ }^{\dagger}$ Cepel \\ Departamento de Redes Elétricas \\ Avenida Horácio Macedo 354, sala 260, \\ CEP 21941-598 - Rio de Janeiro - RJ
}

\section{RESUMO}

Este artigo descreve a concepção de um novo aplicativo computacional (Anatopo) composto por diversos módulos, visando a identificação, diagnóstico e vários tipos de tratamento de configurações topológicas representativas de sistemas de potência descritos por grafos. Tais grafos são definidos por listas de nós (e.g. subestações ou barras), ramos longitudinais (e.g. linhas de transmissão, transformadores, elementos série) e transversais (e.g. cargas, injeções, capacitores e reatores em derivação). O utilitário proposto é útil para operações de pré-processamento de estudos convencionais de fluxo de potência, fluxo de potência ótimo, curtocircuito, confiabilidade, estimação de estado, estabilidade, ou para a avaliação de propriedades estruturais inerentes à topologia de redes.

PALAVRAS-CHAVE: circuito, curto-circuito, fluxo de potência, grafo, mineração de dados, rede, sistema de potência, topologia.

Artigo submetido em 12/10/2008 (Id.: 00903)

Revisado em 28/01/2009, 08/04/2009

Aceito sob recomendação do Editor Associado Prof. Eduardo N. Asada

\section{ABSTRACT}

This paper describes a computer program (Anatopo) useful for dealing with actual large scale power systems described by graphs, defined by a list of nodes (e.g. substations) and branches (e.g. transmission lines). The program have several independent modules which can be applied to a power system, producing a set of diagnoses and topological operations previously selected by the analyst. It has several practical applications related with studies involving the evaluation of power flows, short-circuits, grid structural properties and many others.

KEYWORDS: circuit, short-circuit, power flow, graph, data mining, grid, power system, topology.

\section{INTRODUÇÃO}

Tradicionalmente, os principais esforços evolutivos na análise de sistemas de potência têm se concentrado no desenvolvimento de algoritmos e no aperfeiçoamento de modelos computacionais. Entretanto, um aspecto usualmente relegado a plano secundário situa-se no tratamento dos dados de entrada e dos resultados oriundos das análises realizadas. 
Ocorre porém que, tanto a modelagem quanto as técnicas de simulação, já alcançaram um grau bastante satisfatório de atendimento às necessidades mais comezinhas do usuário típico. Por outro lado, cabe lembrar que, independentemente da precisão de modelos e algoritmos, a qualidade dos resultados obtidos é diretamente influenciada pelo grau de representatividade e coerência dos dados de entrada. Neste contexto vale, portanto, o aforismo: "imprecisão na entrada, erro na saída".

Além disso, observa-se que o crescimento natural dos sistemas de potência reflete-se no conseqüente aumento do conjunto de dados necessários para as análises tradicionais, mormente aqueles representativos da topologia dos sistemas (nós e ramos). Por exemplo, no caso do sistema brasileiro, foi recentemente identificada (situação em 2009) a urgente necessidade de expansão do número total de subestações. Nessa circunstância, tomando-se como referência os utilitários computacionais mais usados no setor elétrico brasileiro, foi necessário aumentar a capacidade de endereçamento das barras, permitindo-se a atribuição de códigos de identificação no intervalo inteiro [1, 99998]. A faixa de codificação anteriormente permitida era restrita ao intervalo [1, 9998]. (Nota: a capacidade de endereçamento não deve ser confundida com o número máximo permissível para as listas de nós e ramos. No caso do sistema brasileiro, são atualmente suficientes listas com 10000 barras e 20000 linhas de transmissão, para estudos de fluxo de potência e até 20000 barras, para estudos de curto-circuito).

Outra questão de interesse refere-se aos agrupamentos de um determinado conjunto de nós sob a denominação genérica de área elétrica. Ocorre que cada nó tem vários atributos de interesse (e.g. empresa proprietária, nível de tensão, região, etc), gerando então dois problemas, quais sejam: (i) a conveniente agregação por classes de atributos; e (ii) a posterior identificação dos ramos de interligação entre as diversas áreas definidas pelos atributos usados. Freqüentemente, deseja-se analisar um sistema à luz de diferentes atributos de área, gerando assim a necessidade de manipulações das diversas áreas, incluindo operações de fusão e extração de conjuntos específicos de nós. O aumento do número de nós reflete-se naturalmente no aumento do número de áreas, também dificultando a manipulação direta das mesmas.

Portanto, o crescimento do sistema praticamente inviabiliza um eventual tratamento manual, com base em inspeção visual por parte do usuário. Isso já é particularmente verdadeiro no caso do sistema elétrico brasileiro hoje existente. Assim sendo, justifica-se plenamente explorar as oportunidades de aperfeiçoamento identificáveis para o tratamento automático de grandes conjuntos dados de entrada e os respectivos resultados dos processamentos computacionais. A manipulação de conjuntos extensos de dados de naturezas diversas, envolvendo a presença de erros, lacunas, incertezas e incongruências, invoca o uso de técnicas computacionais avançadas de mineração de dados, tais como as sugeridas em Do Coutto Filho et alii (2007).

Pari passu com o crescimento dos sistemas, aumenta também a necessidade de prover maior grau de segurança estrutural aos mesmos. Esse tipo de análise não convencional baseia-se no estudo e exploração de propriedades estruturais inerentes à topologia do sistema, consoante Narraway (1993), Macedo et alii (2002), Rosato et alii (2007), Kim e Obah (2007), Kron (1957), Stagg e El-Abiad (1968), Happ (1980).

Os aspectos supracitados respaldam a conveniência de desenvolvimento de uma nova ferramenta computacional, voltada para o tratamento automático de grande volume de dados de entrada e saída, oriundos de estudos convencionais de sistemas de potência. Propõe-se neste artigo um arcabouço conceitual visando este fim, com ênfase particular no tratamento de dados de entrada para estudos de fluxo de potência e curto-circuito. $\mathrm{O}$ aplicativo aqui delineado também poderá ser utilizável em análises envolvendo simulações em tempo real e a preparação de ambientes para estudos de estimação de estado. Nestes casos, é recomendável ou o emprego de padrões abertos de intercâmbio de informações (e.g. modelo CIM - common information model) ou a adaptação dos padrões tradicionais da indústria de energia elétrica (Pierce et alii, 1973).

\section{CONCEITOS BÁSICOS}

Visando uma maior clareza da proposição, justifica-se aqui o enunciado de alguns conceitos ancilares, listados a seguir:

- Barra, Barramento, Nó, Subestação: neste artigo os quatro termos são tomados como sinônimos, por razões de simplicidade (não obstante, podem ser encontradas na literatura definições que indicam diferenças relevantes entre os quatro termos, dependentes do contexto);

- Topologias Origem ou Topologias de Ordem Zero: são os grafos que descrevem as estruturas que se deseja analisar, definidas por uma lista de nós (i.e. barras, subestações) e ramos (i.e. linhas, transformadores, elos de CC, elementos longitudinais e transversais em geral) não submetidos a qualquer tratamento prévio;

- Modo de Operação: vislumbra-se que o aplicativo seja passível de uso sob diferentes modos, tais como, modos pleno, seguro e restrito. Cada um dos mesmos será comentado na seqüência;

- Grau de Hierarquia do Usuário: diz respeito ao nível de autoridade do usuário, no que concerne o modo de operação 
utilizado. No modo de operação pleno exige-se uma senha que confira liberdade total ao usuário (por exemplo, permitese uma operação de renumeração de barras, ignorando completamente a numeração anterior). No modo de operação seguro também exige-se uma senha, porém o grau de liberdade do usuário é mais limitado (por exemplo, o usuário não conseguiria alterar as barras definidas como pétreas, mas teria liberdade para renumerar as demais barras conforme regras desejadas). No modo de operação restrito, embora não haja senha, inibe-se a possibilidade que o usuário faça operações indevidas (por exemplo, preserva-se a denominada topologia matriz, permitindo-se apenas a numeração de barras novas, introduzidas pelo usuário, mas que não pertencem à topologia matriz);

- Barras Pétreas: são as barras que não devem sofrer alteração de atributo a cada processamento do aplicativo, sob os modos de operação seguro e restrito. Sob o modo de operação pleno, os atributos das barras pétreas podem ser alterados (por exemplo, admite-se a atribuição de nova numeração), devendo então ser definido um novo conjunto de barras pétreas (que pode ou não ser vazio);

- Barras Sementes: são barras cujas tensões são de conhecimento seguro do usuário, sendo portanto utilizadas como dados de entrada e reforço de validações. Idealmente, devese dispor de ao menos uma barra semente para cada conjunto de barras isoladas de mesmo nível de tensão, denominadas como ilhas de tensão (por exemplo, se o sistema dispõe de 2 subsistemas em $500 \mathrm{kV}$, interligados apenas por tensões mais baixas, deve-se dispor de um nó semente em cada um desses subsistemas de $500 \mathrm{kV}$ );

- Inicialização: para uma topologia origem, refere-se ao primeiro processamento do aplicativo que deve necessariamente ser no modo pleno, obtendo-se o primeiro conjunto de barras pétreas, se houver. Essa operação é também denominada como batismo;

- I-ésima Topologia-Matriz: após a inicialização, a topologia resultante passa a representar a denominada topologia-matriz de ordem um ou topologia genitora. Os processamentos subseqüentes gerarão as topologias-matrizes de ordem dois, três, etc, da estrutura em análise, também denominadas como topologias descendentes;

- Números Nobres: são aqueles de fácil memorização devido a algum tipo de característica de composição (e.g. dezenas, centenas, milhares, dezenas de milhares, intervalos específicos) ou peculiaridade (77777, etc). Os números nobres podem ser utilizados para identificar as barras pétreas do sistema. Por exemplo, em um sistema de numeração de 5 dígitos (intervalo [1,99998]) dispõe-se dos seguintes números nobres óbvios: 9999 dezenas, 999 centenas, 99 milhares e 9 dezenas de milhares, totalizando 11106 opções para o ba- tismo das barras pétreas). O conjunto de números nobres pode ser definido automaticamente pelo programa ou estabelecido via regras fornecidas pelo usuário;

- Topologia de Referência: é a topologia existente com maior número de nós. Esse conceito é relevante para auxiliar na solução do problema de obtenção de uma numeração simultaneamente compatível para estudos de fluxo de potência e curto-circuito. Seja, por exemplo, a topologia levantada para um certo sistema, visando a realização de estudos de curtocircuito. Suponha-se ainda que a topologia do mesmo sistema para estudos de fluxo de potência apresente um número de barras inferior àquele utilizado na simulação dos estudos de curto. Neste caso, a topologia de referência será aquela usada para o estudo de curto-circuito.

\section{CONCEPÇÃO FUNCIONAL}

\subsection{Dados de Entrada}

O insumo de entrada básico do programa se compõe de três tipos de arquivos: (i) Arquivos do tipo texto, contendo a descrição da topologia de ordem zero. Por exemplo, tomando-se como referência os aplicativos típicos do setor elétrico brasileiro (CEPEL, 2008), o uso de certos comandos específicos gera um arquivo único que contém a lista de nós, ramos e áreas definidos para o sistema em estudo. Propõe-se que o aplicativo a ser desenvolvido seja capaz de tratar até duas topologias, fornecidas como dados de entrada; (ii) Comandos gerais de controle, envolvendo grau de hierarquia do usuário, módulos a serem processados, tipo de processamento, etc; (iii) Arquivos do tipo texto avulsos, em formatações variadas, contendo informações diversas complementares sobre a topologia tratada, como por exemplo, listas de atributos adicionais para nós, ramos e áreas (também denominados como "arquivos-dicionário").

\subsection{Modularidade}

O aplicativo proposto (atualmente em fase de implantação e testes) contém um abrangente conjunto de módulos funcionais, cada um deles abordando um tópico de interesse. Alguns módulos poderão ser invocados de forma independente, enquanto outros deverão ser chamados a atuar de forma concatenada/seqüencial.

O conjunto preliminarmente proposto, em ordem aproximada de interesse prático, engloba os seguintes itens: (i) módulo de identificação, estatística e diagnose; (ii) módulo ordenador; (iii) módulo renumerador simples; (iv) módulo comparador; (v) módulo renumerador duplo; (vi) módulo manipulador de áreas; (vii) módulo de cálculo de métricas; (viii) módulo de ajuste de ponto de operação pré-defeito; (ix) 
módulo matricial; (x) módulo equivalentador; (xi) módulo de relacionamento e renomeação de barras; (xii) módulo configurador.

Do ponto de vista computacional, os módulos são manipulados através um gerenciador, conforme ilustrado na Figura 1. O gerenciador recebe dados de entrada, requisita a operação de um ou mais módulos e fornece os dados de saída. Os dados de entrada incluem não somente as informações topológicas do sistema propriamente ditas, mas também um conjunto de elementos que fornecem subsídios ao processamento de cada módulo, conforme será visto nas seções subseqüentes. De forma similar, os dados de saída podem conter informações diversas, dependendo do tipo de análise ou processamento selecionado.

Os módulos comparador, renumerador duplo e de ajuste solicitam duas topologias como dados de entrada. Nas seções a seguir algumas características funcionais de cada um dos módulos serão apontadas.

\subsection{Identificação, Estatística e Diagnose}

Trata-se de um módulo cujo processamento é sempre invocado, com propósitos de identificação inicial, levantamento estatístico e diagnose da topologia sob análise. A primeira função (identificação) é sempre solicitada, enquanto que as demais têm caráter opcional. Este módulo poderá opcionalmente considerar a materialidade da rede, ou seja, se a topologia tratada é uma rede existente, futura ou histórica. Também podem ser considerados os registros das mutações ocorridas nos elementos (e.g. introdução, retirada e alteração de ramos e nós) durante horizonte de tempo especificado.

1) Identificação: neste âmbito, admitem-se dois enfoques: (i) identificação de atributos nodais; (ii) identificação de atributos ramais longitudinais e transversais (Figura 2). Entre os atributos nodais de interesse podem ser citados:

-Categorias nodais diversas: no sistema brasileiro, os nós da rede podem ser enquadrados nas categorias de rede básica (tensões iguais ou superiores a $230 \mathrm{kV}$ ) e rede não-básica (tensões inferiores a $230 \mathrm{kV}$ ).

Também podem ser reconhecidos barramentos seccionadores, dos quais partem apenas ramos que são linhas de transmisão. Da mesma forma também podem ser distingüidos barramentos transformadores (dos quais partem apenas transformadores) e barramentos híbridos (conectados a transformadores e linhas). Outra categoria nodal de interesse se refere à natureza das injeções de barras, quais sejam: barra de carga (consumidores livre, industrial, comercial, residencial), barra de geração (térmica, hidráulica, nuclear, eólica, etc), barra de carga e geração, barra com derivação reativa

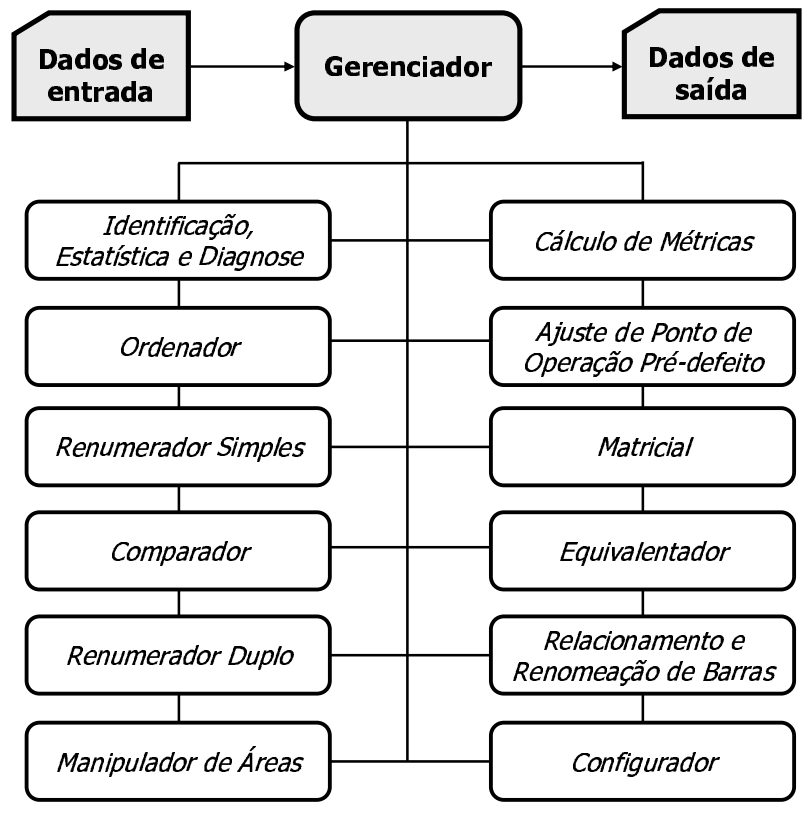

Figura 1: Estrutura Computacional

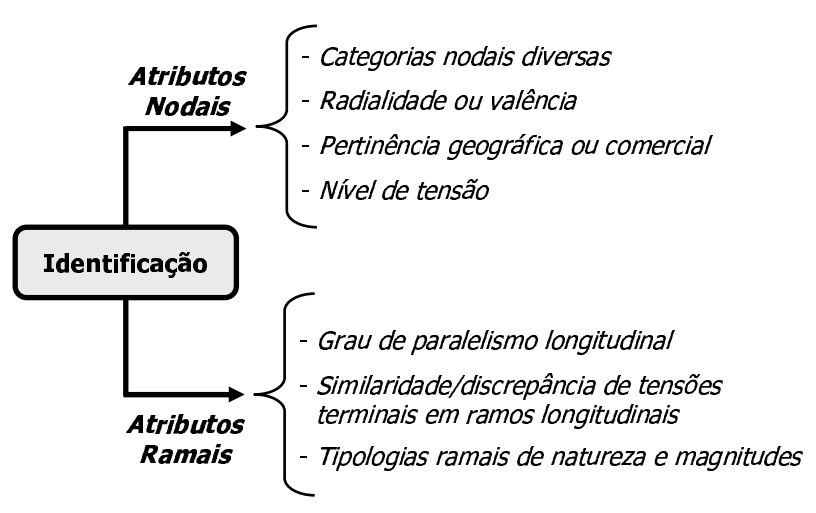

Figura 2: Formas de Identificação Topológica

(capacitor, reator), barras CA, barras CC, etc. Em determinados estudos específicos, podem ser geradas novas categorias de interesse, onde os barramentos estão relacionados a certos tipos de redes. É o caso, por exemplo, das redes definidas para o Sistema Interligado Nacional (ONS, 2008): rede de operação, rede de simulação, rede de observação, rede básica.

Algumas categorias nodais podem apresentar grandes dificuldades para uma identificação automática sem auxílio do usuário (por exemplo, via arquivos externos), embora sejam relevantes em vários contextos. Por exemplo, são de grande interesse classificações de barras como as seguintes: real, fictícia, auxiliar, tape, seccionadora, primária, secundária, terciária, barra de mútua, barra de transformador elevador, barras de capacitores e reatores série, barras terminais de gera- 
dores, etc.

Finalmente, os tipos de ramos transversais e logitudinais conectados a um barramento podem ainda auxiliar na identificação de características de interesse, suscitando então categorias de tipos de conexão (por formatação ou por ordem de grandeza numérica) tais como os genericamente exemplificados na Tabela 1. Esse tipo de levantamento também pode ser útil quando da busca da presença de erros de modelagem ou tipificação dos elementos conectados. Por exemplo, para um dado nó, a presença exclusiva de grandezas no formato $+R+j X$ conectadas ao mesmo, pode sugerir que os ramos incidentes são linhas de transmissão. Por outro lado, valores longitudinais no formato $-j X$ podem sugerir a existência de transformadores de 3 enrolamentos representados na configuração em estrela ou capacitores série. Valores elevados de $+j X$ podem indicar a incidência de cabos subterrâneos e certas faixas de valores $\pm R \pm j X$, tanto longitudinais quanto transversais, podem, em situações particulares, auxiliar na identificação de elementos FACTS conectados ao nó em apreço, ou a eventual modelagem de transformadores de 3 enrolamentos na configuração delta (Westinghouse, 1964), etc.

Tabela 1: Tipos de Ramos Incidentes em um Nó

\begin{tabular}{|c|c|}
\hline Lado Jusante $^{*}$ & Lado Montante $^{*}$ \\
\hline $\pm R \pm j X$ & $\pm R \pm j X$ \\
\hline $\pm R \pm j X$ & $\pm j X$ \\
\hline $\pm R \pm j X$ & $\pm R$ \\
\hline $\pm j X$ & $\pm j X$ \\
\hline $\pm R$ & $\pm R$ \\
\hline $\pm R$ & $\pm j X$ \\
\hline
\end{tabular}

(*) Arbitrariamente definido

-Radialidade ou valência nodal (i.e. número de ramos conectados ao nó): esse atributo pode ajudar a identificar barras críticas do sistema sob o ponto de vista de segurança estrutural, como também auxiliar a detecção de barras fictícias associadas a transformadores de 3 enrolamentos (e.g. uma valência igual a 3 associada à presença de um ramo com reatância negativa pode ser um indicativo da presença de um transformador de 3 enrolamentos).

-Pertinência geográfica ou comercial: esse atributo diz respeito à localização espacial (país, estado, região, área, instalação) ou à propriedade do barramento (empresa). Sua identificação automática pode ser viabilizada com o auxílio de arquivo externo à guisa de "dicionário". A título de exemplo, observa-se que uma identificação preliminar e parcial de barramentos por estado pode ser alcançada pela identificação dos códigos das áreas elétricas às quais esses barramen- tos pertencem, tal como usualmente adotado no setor elétrico brasileiro. Isso se dá porque em muitas situações uma certa empresa atua em um único estado da federação.

-Nível de tensão do nó: este atributo é de grande relevância e deverá ser passível de tratamento por cinco estratégias distintas, a saber (Figura 3):

a) Identificação via caracteres do nome da barra: essa metodologia poderá ser implementada de forma ingênua (e.g. pela simples pesquisa dos 3 últimos campos do nome da barra) ou de forma mais elaborada, através da pesquisa de diferentes combinações de conjuntos de posições e aglutinações de campos do nome da barra. A estratégia elaborada poderá opcionalmente fundamentar-se em técnicas de inferência neuro-nebulosas (Ross, 1995).

b) Identificação via "dicionário de barras": nesse caso, o levantamento da tensão desconhecida é feita procurando-se o código numérico da barra pesquisada em um "dicionário" (i.e. lista registrada em arquivo independente) de barras supostamente disponível ao usuário. Nessa opção podem ocorrer situações nas quais o código numérico é identificado no dicionário, mas o nome da barra pesquisada difere do nome registrado no dicionário. $\mathrm{O}$ grau de credibilidade dado ao dicionário é decidido pelo usuário, podendo ser estabelecido por escala numérica. Embora esse grau de confiança atribuído ao dicionário possa ser bastante acentuado, esse método apresenta a desvantagem de exigir a constante manutenção e atualização do próprio dicionário.

c) Identificação via código intrínseco opcional da lista investigada: é possível que a própria lista de barras, cujas tensões se deseja identificar, já contenha informações sobre essas mesmas tensões. Por exemplo, a lista de barras pode conter um campo já com o valor nominal da tensão da barra ou ainda com um código que identifique um determinado grupo base de tensão ao qual aquela barra pertence. Nesse caso, a identificação é imediata e direta. Não obstante, também é comum a omissão parcial ou mesmo total desse campo e também o registro errôneo do campo (o grupo base de tensão indica uma tensão diferente da tensão nominal da barra). O grau de credibilidade desse campo também pode ser opcionalmente definido pelo usuário.

d) Método da conectividade coerente de tensões: esse método parte do pressuposto que barras com tensões idênticas são conectadas por linhas de transmissão, enquanto barras com tensões diferentes ligam-se por transformadores. Sendo assim, o método depende da identificação prévia da natureza de todos os ramos da topologia sob análise (i.e. quais ramos são linhas e quais ramos representam transformadores). Nota-se que essa premissa garantiria apenas que certos conjuntos de barras devem ter a mesma tensão, porém o simples mapeamento de todas as conexões não é suficiente para a de- 


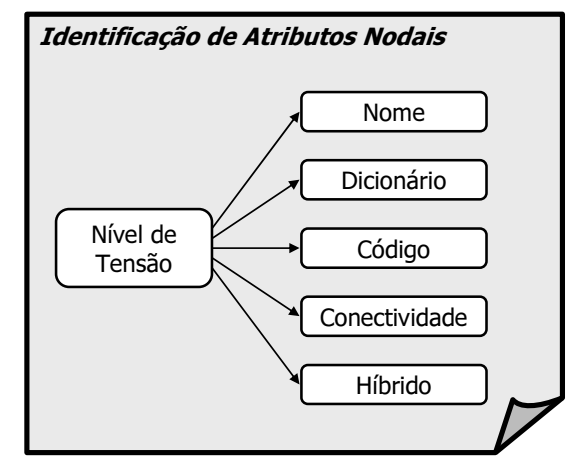

Figura 3: Métodos de Identificação do Nível de Tensão

terminação do valor numérico das tensões. Entretanto, para cada conjunto de barras identificado como tendo a mesma tensão, esse problema pode ser resolvido se for informada a tensão de uma única barra, definida como "barra semente" (vide Seção II).

e) Método híbrido: trata-se aqui do uso combinado das potencialidades de todos os métodos anteriores, amparado num sistema de ponderação hierárquica de credibilidade (por exemplo, votação ponderada por pesos definidos pelo usuário) e sistema de denúncia e filtragem das discrepâncias detectadas.

Entre os possíveis atributos ramais, têm potencial interesse os seguintes:

-Grau de paralelismo longitudinal: esse atributo aponta o número de circuitos em paralelo entre barras conectadas pelos mesmos.

- Similaridade/discrepância de tensões terminais em ramos longitudinais: esse é um atributo auxiliar para a detecção de patologias de modelagem eventualmente presentes no sistema. Por exemplo, a identidade de tensões terminais reforça a possibilidade do ramo ser uma linha de transmissão. Por outro lado, a discrepância dessas tensões aponta a possível presença ou de um transformador, ou a conexão a uma barra especial (barra de geração com tensão desconhecida, barra auxiliar fictícia, etc).

-Tipologias ramais de natureza e magnitudes: trata-se aqui da identificação das naturezas dos ramos, quais sejam, linhas de transmissão, interligações, transformadores de 2 ou 3 enrolamentos de malha, de fronteira ou de distribuição, capacitores e reatores série e em derivação, transformadores com resistência não nula, condutâncias de dispersão, elementos ramais fictícios, linhas aéreas, cabos subterrâneos, transmissão CA/CC, equipamentos FACTS, etc. Admite-se aqui também uma classificação por faixas de magnitudes paramétricas das grandezas $\pm \mathrm{R} \pm \mathrm{jX}, \pm \mathrm{G} \pm \mathrm{jB}$ e a relação $\mathrm{X} / \mathrm{R}$ (vide
Tabela 1).

2) Levantamento estatístico: neste âmbito cabem miríades de contabilizações de interesse. Entre estas, pode-se, a título de exemplo, citar diversas totalizações automáticas de barras e/ou ramos, com ou sem qualificativos, contagem de barras e/ou sistemas isolados por nível de tensão ou sem conexão elétrica, contabilizações de atributos diversos especificados opcionalmente pelo usuário (e.g. carga total, número de geradores, reatâncias negativas, identificação de links, contagem do número de malhas topológicas, interligações, ramos com características radiais, etc).

3) Diagnose: enquadram-se aqui, em caráter opcional, controlado pelo usuário, distintos indicadores topológicos, tais como histogramas de relações X/R e indicadores topológicos de vulnerabilidade como os mencionados em Rosato et alii (2007) e Kim e Obah (2007). Também podem ser aqui propostos indicadores diagnosticando o grau de capilaridade ou radialidade de um sistema (i.e. índices que auxiliem na diferenciação entre sistemas densamente malhados daqueles com características mais radiais). Esta função tem caráter opcional.

\subsection{Ordenação}

Este módulo tem por objetivo reordenar, por ordem numérica dos códigos de barra (de forma crescente ou decrescente) as posições de uma lista de nós de uma dada topologia, segundo regras estabelecidas pelo usuário. As duas técnicas propostas são mencionadas a seguir.

A reordenação simples opera apenas o remanejamento ordenado de posições levando em conta tão-somente a numeração das barras.

A reordenação qualificada promove uma ordenação de posições compondo a numeração das barras com outros atributos especificados pelo usuário (e.g. níveis de tensão, área elétrica, etc). É claro que a reordenação qualificada só pode ser processada quando os atributos já tiverem sido informados (via arquivo externo) ou levantados por outro módulo conveniente.

Este módulo é de uso opcional e pode ser invocado quando a topologia de ordem zero apresenta-se com algum grau de desordem ou quando o usuário deseja certificar-se de que não há barras com o mesmo código numérico de identificação. $\mathrm{O}$ módulo ordenador também facilita a obtenção de uma matriz incidência convenientemente montada (i.e. ordenada), que pode ser posteriormente submetida ao módulo comparador, visando a detecção de discrepâncias. Quando a ordenação qualificada é processada, obtêm-se informações necessárias para a montagem de diferentes tipos de equivalentes estáti- 
cos, definidos pelo usuário (Klos e Borkowska, 1979; Schilling, 1979; Aitchison e Klos, 1991).

\subsection{Renumeração Simples}

Para uma topologia inicial, denominada topologia-matriz de ordem zero (vide Seção II), este módulo promove a primeira numeração (para o caso em que as barras ainda não dispõem de números, operação batismo) ou a primeira renumeração completa ou parcial, consoante regras previamente selecionadas pelo usuário e em consonância com o modo de operação definido (pleno, seguro e restrito, vide Seção II). Seu processamento é opcional. Considerando, por exemplo, um modo de operação em 5 dígitos, este módulo aponta uma seleção de números no intervalo [1,99998] a serem atribuídos aos nós do sistema. A atribuição é realizada segundo uma prioridade de regras definidas pelo usuário, porém preservando uma hierarquia conveniente para o uso dos números nobres, de fácil memorização (i.e. dezenas, centenas, milhares, etc) e preservando a numeração das barras definidas como pétreas (vide Seção II).

A atribuição automática de números a uma lista de barramentos pode ser realizada mediante diversas estratégias, em diferentes graus de sofisticação, com e sem a intervenção do usuário. Entre estas, cabem ser citadas a seguintes, previstas para o aplicativo:

1) Numeração seqüencial simples: neste caso, o primeiro número pode ser opcionalmente informado pelo usuário. A numeração pode ser feita com ou sem intervalos. Uma alternativa baseia-se na definição do primeiro número e o intervalo a ser usado. Outra possibilidade procura utilizar o máximo intervalo possível (dentro de uma faixa arbitrária) para o conjunto de barras sob tratamento. Variantes dessa estratégia podem tomar como referência o último número, definido pelo usuário. Embora essa metodologia seja muito simples, ela não permite a retenção de informações particulares associadas aos números.

2) Numeração aleatória: essa metodologia produz uma numeração totalmente sem controle por parte do usuário, que uma vez processada poderá ser submetida ao módulo ordenador. Ver-se-á então que os intervalos entre números não obedecem a qualquer regra determinística. Esse tipo de numeração pode ser útil para fins de operações intermediárias de validação de outros módulos aqui discutidos, visando a filtragem de erros.

3) Numeração por hierarquia de agregados: essa estratégia solicita inicialmente a definição dos tipos de agregados que interessam (por exemplo, níveis de tensão e regiões geográficas). Uma vez estabelecidos os tipos de agregados a serem considerados, há que se definir a hierarquia de pre- cedência que deve vigorar entre os mesmos. No exemplo em questão supor-se-á que interessa a definição de 3 níveis de tensão $(A, B, C)$ e 2 regiões geográficas $(x, y)$, possibilitando a composição de $3 \times 2=6$ conjuntos de agregados do tipo tensão/região (Ax, Ay, Bx, By, Cx, Cy). Ainda a título de exemplo, será suposto que a tensão tem precedência hierárquica sobre a região. Na seqüência, avalia-se o porte de cada um dos agregados, e procede-se à alocação proporcional dos números, consoante o tamanho da cada conjunto e de acordo com a regra de atribuição selecionada. No exemplo tratado será suposto que cada um dos seis agregados apontados tem, respectivamente, $9,4,8,7,2$ e 5 barramentos. Se, por exemplo, a regra de atribuição selecionada impõe o uso de números nobres da faixa dos milhares e um intervalo fixo de largura 2, um possível resultado da operação de batismo seria:

Agregado Ax - 1000, 1002, 1004, 1006, 1008, 1010, 1012, 1014, 1016

Agregado Ay - 1018, 1020, 1022, 1024

Agregado Bx - 2000, 2002, 2004, 2006, 2008, 2010, 2012, 2014

Agregado By - 2016, 2018, 2020, 2022, 2024, 2026, 2028

Agregado Cx - 3000, 3002

Agregado Cy - 3004, 3006, 3008, 3010, 3012

No exemplo acima, dependendo das regras de atribuição selecionadas, diversos outros resultados seriam possíveis. Poder-se-ia, por exemplo, impor uma diferenciação mais acentuada para as barras de mesma tensão mas de regiões distintas, através de um intervalo discriminador específico ou pelo uso de números nobres atrelados às combinações de milhares e centenas. Por exemplo, os dois primeiros agregados da tensão A e regiões x e y ficariam:

Agregado Ax - 1000, 1002, 1004, 1006, 1008, 1010, 1012, 1014, 1016

Agregado Ay - 1100, 1102, 1104, 1106

Entre as diversas categorias de agregados de interesse, as seguintes merecem menção:

- conectividades (i.e. identificando sistemas isolados);

- níveis de tensão (maior tensão, menor tensão, rede básica);

- pertinências geográfica (país, regiões, estados, áreas);

- pertinências comerciais;

- tipos de estudos (fluxo de potência, curto-circuito, estabilidade).

Cumpre ainda enfatizar que a atribuição de números pode depender inicialmente da contabilização dos diferentes agre- 
gados e da verificação da grandeza (i.e. porte) desses agregados. Assim, para uma faixa de números permitidos, opcionalmente especificada pelo usuário, a atribuição de números aos agregados pode ocorrer por:

a) alocação seqüencial (direta sem intervalos, porém respeitando a hierarquia definida, direta com intervalos, por ordem de grandeza usando números nobres, tais como centenas, milhares, dezenas de milhares, etc, tal como no exemplo acima);

b) alocação proporcional ao tamanho dos agregados (com ou sem intervalos separadores, usando ou não números nobres);

c) alocação híbrida, pois também pode haver necessidade da consideração de regras especiais visando o tratamento de tópicos relevantes tais como: a presença de barramentos de geração para os quais se deseje reservar números mnemônicos, atribuíveis a nós representativos de terminais de geradores e transformadores elevadores, a presença de eventuais barras pétreas, facilidade de identificação de barras auxiliares, fictícias, secundárias, terciárias, derivações, mútuas, seccionadoras, etc.

d) listas de regras ad-hoc definidas pelo usuário (e.g. reservas de intervalos, etc).

\subsection{Comparador}

Este módulo compara duas topologias, podendo operar sob dois enfoques distintos e independentes: (i) comparador de estrutura topológica: visa identificar o grau de similaridade estritamente estrutural entre duas topologias às quais foi aplicada uma mesma regra de ordenação. Dado que a mesma regra de ordenação foi previamente aplicada às duas estruturas (vide módulo ordenador), espera-se que a análise das respectivas matrizes incidências possa caracterizar o grau de coincidência topológica entre as duas estruturas. Observase que as duas topologias podem representar sistemas completamente distintos, porém com estruturas topológicas total ou parcialmente semelhantes. Nesse caso, um possível indicador de similaridade estritamente topológica é dado pela sequiência de nós e ramos definidores de cada malha do sistema. Um outro indicador ingênuo poderá também ser extraído da tentativa de superposição direta das duas matrizes, aliada à conseqüente evidência das discrepâncias. Essas discrepâncias podem ser tentativamente minimizadas pela busca de uma ordenação otimizada heuristicamente. Outros possíveis indicadores ingênuos podem ainda basear-se na simples contagem dos nós, ramos e malhas de ambas as topologias. Nessa forma de operação, o aplicativo gera listas de supostas congruências, que poderão ser validadas ou não pelo ususário; (ii) identificador de coincidências: visa a detecção de conjuntos de nós e ramos comuns entre duas topologias que representam pretensamente o mesmo sistema. A utilidade deste modo de operação é a identificação da lista de nós e ramos coincidentes para fins de posteriorior renumeração compatibilizada (e.g. no caso de topologias usadas em estudos de curto-circuito e fluxo de potência).

O identificador de coincidências admite operação semiautomática ou com a intervenção parcial do usuário. No primeiro caso, o tratamento baseia-se tão somente na detecção de coincidências existentes nas matrizes incidência, previamente montadas com base no mesmo critério de ordenação. No segundo caso, o usuário fornece uma lista reduzida de barras coincidentes nas duas topologias. Com base nessa lista e nas matrizes incidência ordenadas pelo mesmo critério, o comparador emite então um diagnóstico relacionando a lista de barras idênticas nas duas topologias, as listas de barras existentes em uma única topologia, a lista de ramos existentes em ambas as topologias, os ramos presentes em uma única topologia e um diagnóstico das discrepâncias ramais sob o ponto de vista paramétrico, com o respectivo percentual de erro relativo em relação à topologia tomada como referência. Para as barras coincidentes, gera-se também um diagnóstico de similaridades e discrepâncias das grandezas em derivação (injeções, cargas, capacitores, reatores, etc). Esse módulo deve viabilizar a identificação de barras eliminadas ou introduzidas em relação à topologia de referência (vide Seção II) e também a comparação de listas de barras qualificadas por atributos. É relevante enfatizar que a comparação aqui realizada não se baseia nos números atribuídos a cada barramento, mas unicamente ao aspecto estrutural topológico.

\subsection{Renumeração Dupla}

Este módulo renumera duas topologias previamente submetidas ao módulo comparador, operado na forma de identificador de coincidências, tomando uma delas como referência (vide Seção II) para a seleção de novos números, consoante regras estabelecidas pelo usuário. Trata-se de uma funcionalidade útil para a compatibilização da numeração de barras de topologias oriundas de estudos de curto-circuito e fluxo de potência.

\subsection{Manipulação de Áreas}

Este módulo tem por objetivo operar três etapas consecutivas, quais sejam: (i) diagnose inicial; (ii) manipulações; (iii) diagnose final. As etapas inicial e final visam produzir tabelas de quatro colunas, tais como ilustrado na Tabela 2, nas quais é possível identificar como as áreas do sistema estão conectadas entre si, antes e após a operação de manipulação intermediária. As diagnoses podem incluir, entre outras, a 
contabilização e o controle dos códigos numéricos de área utilizados, os códigos ainda livres e diversas estatísticas qualificadas por atributos de interesse (e.g. quantas interligações existem entre áreas, num dado nível de tensão, qual o tipo de interligação, transformador ou linha, etc).

Tabela 2: Diagnoses Inicial e Final de Áreas

\begin{tabular}{|c|c|c|c|c|}
\hline \multirow{2}{*}{$\begin{array}{c}\text { Número } \\
\text { da Área }\end{array}$} & Nome & Áreas com as & \multicolumn{2}{|c|}{$\begin{array}{c}\text { Lista de Ramos que } \\
\text { da Área }\end{array}$} \\
& & quais se Conecta & \multicolumn{2}{|c|}{ Interligam as Áreas } \\
\cline { 4 - 5 } & & & Da Barra & Para Barra \\
\hline
\end{tabular}

A operação intermediária (a manipulação propriamente dita) compreende as seguintes alternativas, opcionalmente invocadas pelo usuário: (i) fusões e desmembramentos generalizados; (ii) delimitações específicas; (iii) caracterização por estado da federação.

A operação de fusão resulta numa situação na qual o número de áreas final pode ser inferior ao número de áreas inicial. Essa manipulação pode ser viabilizada fazendo-se:

- acesso à lista de áreas a serem fundidas;

- atualização de nome e número da nova área resultante da fusão;

- marcação de todas as barras da nova área resultante.

Em contraposição, a operação de desmembramento pode gerar uma configuração na qual o número de áreas final pode ser superior ao número de áreas inicial, contemplando a criação de áreas completamente novas. A título de esclarecimento, suponha-se que as novas áreas a serem criadas sejam denominadas como "segmentos". Assim a operação desmembramento pode ser sucintamente viabilizada fazendo-se:

- acesso à lista de segmentos;

- para um dado segmento definido, atualização do conjunto de barras desmembradas de áreas pré-existentes;

- promove, se for o caso, a eventual agregação de barras isoladas ao segmento sob construção.

A delimitação específica é uma técnica que pode revelar-se conveniente na situação em que a manipulação de barras na composição de áreas ocorre de forma muito particularizada, exigindo o uso de arquivo externo com as informações de interesse.

A caracterização por estado nada mais é do que um caso particular de delimitação específica. Trata-se de uma operação de difícil automatização, viabilizando-se, porém, via arquivo externo.

\subsection{Cálculo de Métricas}

Dadas duas topologias ou dois pontos de operação associados a uma dada topologia, esse módulo viabiliza o cálculo de métricas diversas representativas de "distâncias" topológicas, "distâncias" entre pontos de operação, graus de discrepâncias paramétricas ou a combinação dos três fatores. Seu uso é opcional.

\subsection{Ajuste de Ponto de Operação}

Para uma topologia conhecida, esse módulo promove o estabelecimento automático do ponto de operação pré-distúrbio mais realista possível, com base em algumas informações oriundas de medições e registros do histórico operativo da topologia em questão. Atua minimizando a "distância" entre os pontos de operação (ponto de operação medido e ponto de operação ajustado), utilizando o módulo de cálculo de métricas. Trata-se de um módulo útil para a obtenção de pontos de operação pré-distúrbio, visando a simulação dinâmica de perturbações ocorridas no sistema.

\subsection{Módulo Matricial}

Para uma dada topologia, este módulo opera um conjunto de funções matriciais, opcionalmente solicitadas pelo usuário, entre as quais cabe citar: montagem de matrizes incidência e primitivas, montagem das matrizes admitância e impedância nos referenciais de nó, malha, ramo e híbrido (Shipley, 1976), autovalores, traços e determinantes, cálculo de atributos topológicos (celularidade, planaridade, grau de esparsidade, radialidade nodal, grau de capilaridade, contagem do número de ilhas por nível de tensão, contagem de sistemas isolados). O módulo contém ainda recursos para o tratamento de matrizes por técnicas de esparsidade (Brameller, 1976; Morozowski Filho, 1981).

\subsection{Módulo Equivalentador}

Viabiliza a contração via redução de Kron (Kron, 1957; Brown, 1975) e a transfiguração (Klos e Borkowska, 1979; Schilling, 1979; Aitchison e Klos, 1991) de uma dada topologia (equivalente-árvore). É útil para estudos de fluxo de potência e curto-circuito (eliminação de mútuas). 


\subsection{Relacionamento e Renomeação No- dal}

É um módulo que faz a relação entre nomes e números de duas listas distintas ou promove a atribuição de novos nomes às barras do sistema, consoante um conjunto de regras definidas pelo usuário. A primeira função é útil na situação na qual duas empresas diferentes identificam o mesmo sistema de forma diferenciada, como ilustrado simbolicamente no esquema da Tabela 3. Essa relação nada mais é do que uma espécie de "tradutor" que interpreta a nomenclatura de barras e códigos numéricos usados por empresas que eventualmente não abrem mão de suas codificações internas (nome e número de barra), em prol de uma uniformidade de representação. A segunda função promove, em uma das listas, a alteração de nomes e/ou números das barras comuns a ambas as listas, tomando a outra das listas como paradigma. Obviamente, a alteração conjunta de nome e número resultará numa única lista, caso os dois sistemas representados sejam topologicamente idênticos.

Tabela 3: Tradutor de Barras Representativas de um Mesmo Sistema

\begin{tabular}{|c|c|c|c|}
\hline \multicolumn{2}{|c|}{ Empresa A } & \multicolumn{2}{c|}{ Empresa B } \\
\hline lista de & lista de & lista de & lista de \\
números $A$ & nomes $A$ & números $B$ & nomes $B$ \\
\hline
\end{tabular}

\subsection{Configurador}

A principal função do módulo configurador é auxiliar na montagem de topologias nas quais se deseja a representação discriminada de chaves e disjuntores, necessária em estudos de estimação de estado, análise em tempo-real e modelagem de subestações em estudos de confiabilidade. Como é sabido, não é possível obter-se uma configuração topológica discriminando-se chaves e disjuntores, a partir, tão-somente, de uma configuração descritora de barras e ligações (i.e. ramos). Para que isso seja factível, são necessárias informações adicionais, definidoras dos arranjos físicos das subestações. Por outro lado, a recíproca não ocorre, ou seja, é possível a obtenção de configurações barra/ligação, a partir de configurações chave/disjuntor.

\section{CONCLUSÕES}

Este artigo apresentou a concepção de um novo aplicativo computacional, útil para o tratamento de sistemas elétricos de grande porte, cuja representação topológica não admite um tratamento manual eficiente. A principal contribuição da proposta reside na metodologia inédita de identificação de congruências nodais de topologias de grande porte, baseada em ordenação prévia de matrizes incidência.

\section{AGRADECIMENTOS}

Esse trabalho foi parcialmente viabilizado graças ao apoio do Cepel, ONS, CNPq, CAPES e FAPERJ. Em particular, registra-se o incentivo prestado pelos Eng. Marcelos G.dos Santos (ONS), Eng. Flávio F. Pazo Blanco (ONS), Dr. Flávio Rodrigo Miranda Alves (CEPEL), Dr. Sergio Porto Romero (CEPEL), Dr. Gilberto Pires de Azevedo (CEPEL). Os autores também agradecem pelas contribuições dos revisores e do editor da revista Controle \& Automação.

\section{REFERÊNCIAS}

Aitchison, P.W. and Klos A.(August 1991). Derivation of Equivalent Networks. Int. J. of Electrical Power \& Energy Systems, Vol.13, No.4, pp.209-215.

Brameller, A. (1976). Sparsity: Its Practical Applications to Systems Analysis. Pitman, London.

Brown, H.E (1975). Solution of Large Networks by Matrix Methods. John Wiley \& Sons, Inc., New York.

CEPEL (2008). Programa de Análise de Redes - Manual do Usuário Versão 09.04.03. Rio de Janeiro, Dezembro.

Do Coutto Filho M.B., Schilling M.T.e Souza J.C.S. (Jan./Mar.2007). Sobre o Problema da Integração Generalizada de Dados. Controle \& Automação, SBA, Vol. 18, no. 1, pp. 24-43.

Happ, H.H (1980). Piecewise Methods and Applications to Power Systems. John Wiley \& Sons, New York.

Kim, C.J. and Obah O.B. (2007). Vulnerability Assessment of Power Grid Using Graph Topological Indices. Int. J. of Emerging Electric Power Systems, Vol. 8, Issue 6, art.4, pp. 1-15(on-line).

Klos A. and Borkowska, B. (1979). Equivalent Transfiguration of Networks. Int. J. of Electrical Power \& Energy Systems, Vol.1, No.1, pp. 30-34.

Kron, G.(1957). Diakoptics: Piecewise Solution of LargeScale Systems. General Engineering Laboratory, General Electric Co, Schenectady, USA.

Macedo Jr., J.R.; Resende, J.W. and Samesima, M.I. (2002). The Inherent Structure Theory of Networks and Admittance Matrix Sparsity Relationship. in Proc. of 10th. Int. Conf. on Harmonics and Quality of Power, Vol.1, pp.127-131.

Morozowski Filho, M. (1981). Matrizes Esparsas em Redes de Potência: Técnicas de Operação. Livros Técnicos e Científicos Editora, Rio de Janeiro. 
Narraway, J.J.(October 1993). Probability, Graphs and Electrical Networks. IEE Proc.-G, Vol.140, No.5, pp.347354.

ONS (2008). Procedimentos de Rede - Módulo 1, Submódulo 1.1. (disponível em www.ons.org.br).

Pierce Jr. H. E-Chairman et alii (November 1973), Working Group on a Common Format for Exchange of Solved Load Flow Data. Common Format for Exchange of Solved Load Flow Data. IEEE Trans. on PAS, Vol. PAS-02, No. 6, pp.1916-1925.

Rosato, V., Bologna, S. and Tiriticco, F. (2007). Topological Properties of High-Voltage Electrical Transmision Networks. Electric Power Systems Research, Vol. 77, pp.99-105.

Ross, T.J. (1995). Fuzzy Logic with Engineering Applications. McGraw-Hill, Inc., New York.

Schilling, M.T. (1979). Métodos de Eqüivalência em Sistemas de Energia Elétrica. Dissertação de M.Sc., Programa de Engenharia Elétrica, COPPE/UFRJ, Rio de Janeiro.

Shipley, R.B. (1976). Introduction to Matrices and Power Systems. John Wiley \& Sons, New York.

Stagg, W.G.and El-Abiad, A.H.(1968). Computer Methods in Power Systems Analysis. Caps. 3 e 4, McGraw-Hill Book Company, New York.

Westinghouse Electric Co. (1964). Electrical Transmission and Distribution Reference Book. 4 th. Edition, Cap. 2, Pittsburgh, USA. 